spillage increases exposure risk.

This protocol has several benefits. First, it can dramatically reduce the healthcare workers' exposure to high-risk body fluids. Second, because the cost of a two-piece, closed-end pouch is significantly less than that of a one-piece drainable pouch, it could result in a cost savings to the facility. There are Medicare benefits as well. Many carriers do not limit monthly usage of closed-end pouches, thereby assisting in alleviating increased financial burden to the facility. The use of closed-end pouches in the various healthcare settings provides a cost-effective avenue for facilitating compliance to OSHA standards.

\section{CDC Grants}

'Equivalency' to 22

State Guidelines for

Management of HIVand HBV-Infected Healthcare Workers

by Gina Pugliese, RN, MS Medical News Editor

October 28, 1992, marked the deadline for state public health officials to submit to CDC their guidelines for managing HIV and hepatitis B-infected healthcare workers performing exposure- prone invasive procedures. All 59 states and territories have complied with the federal law (Section 663 of Public Law 102-141) requiring the adoption, as written, of the July 12,1991 , CDC "Recommendations for Preventing Transmission of HIV and HBV to Patients During Exposure-Prone Invasive Procedures"; certification that they are equivalent; or request for a one-year extension.

Eight states have adopted the CDC guidelines as written. The 22 states that requested their guidelines to be certified as equivalent to the July 12, 1991, CDC guide- lines were granted "equivalency." Twenty-eight states were granted a one-year extension, and certification is pending in one state.

The CDC has allowed for a broad interpretation of its guidelines, granting equivalency, for example, to those guidelines that include a "case-by-case" evaluation of HIV - and HBV-infected healthcare workers performing exposure-prone procedures, voluntary testing of healthcare workers, and confidentiality regarding the infection status of any healthcare worker who is determined to be fit for duty. 